\title{
On the size of the smallest scales in cosmic string networks
}

\author{
Xavier Siemens, Ken D. Olum and Alexander Vilenkin \\ Institute of Cosmology \\ Department of Physics and Astronomy \\ Tufts University \\ Medford MA 02155, USA
}

(October 30, 2018)

\begin{abstract}
We present a method for the calculation of the gravitational back reaction cutoff on the smallest scales of cosmic string networks taking into account that not all modes on strings interact with all other modes. This results in a small scale structure cutoff that is sensitive to the initial spectrum of perturbations present on strings. From a simple model, we compute the cutoffs in radiation- and matter-dominated universes.
\end{abstract}

PACS numbers: $11.27 .+\mathrm{d}, 98.80 . \mathrm{Cq}$

\section{INTRODUCTION}

Topological defects are a generic prediction of grand unified theories. They can be formed in phase transitions when the topology of the vacuum manifold of the low energy theory is non-trivial [1]. For a review see [2].

The result of a phase transition that produces strings is a network of long strings that stretch across the horizon and a collection of closed loops. If the phase transition occurs at energy $\eta_{s}$, then the mass per unit length in the strings is $\mu \sim \eta_{s}^{2}$. Immediately after formation, strings in the early universe undergo an epoch of heavy damping until a time $t_{*}$, which depends on the string formation scale $\eta_{s}$ as well as the specifics of the particle physics model that produces them. As a result of this damping all sub-horizon features, such as loops and perturbations on long strings, are wiped out and the long strings and loops are Brownian with a persistence length $d \sim t_{*}$.

After that time, numerical simulations show that in an expanding universe there is an attractor solution in which the network evolves into a "scaling regime" (see [2] and references therein), where the energy density of the string network is a small constant fraction of the radiation or matter density and the statistical properties of the system, such as the correlation lengths of long strings and average sizes of loops, scale with the cosmic time $t$. This solution is possible because of intercommutations that produce cosmic string loops which in turn decay by radiating gravitationally. Simulations also have found that most loops and the perturbations on long strings have the smallest possible size, the simulation resolution, which does not scale. The prevailing opinion on this issue is that the size of small-scale structure in fact also scales with the cosmic time $t$ and its value is given by the gravitational back-reaction scale $\Gamma G \mu t$, where $\Gamma$ is a number of order 100 and $G$ is Newton's constant. This possibility was first pointed out in [3].

Cosmic strings are good candidates for a variety of interesting cosmological phenomena such as gamma ray bursts [4], gravitational waves [5, 6] and ultra high energy cosmic rays [7, 8]. Some of the predictions of these mod- els, however, depend sensitively on the so far unresolved question of the size of the small-scale structure.

\section{BACK-REACTION MODEL}

Hindmarsh [9] (see also [10]) showed that the power per unit length radiated into gravitational waves from two colliding arbitrary small perturbations of long repeat length $L$ on an infinite string is

$$
\frac{d P}{d l} \sim \pi G \mu^{2} \sum_{n, m}\left(\kappa_{n}+\kappa_{m}\right) \epsilon_{n}^{2} \epsilon_{m}^{2}
$$

where $\kappa_{n}=2 \pi n / L$ and $\kappa_{m}=2 \pi m / L$ are the wavenumbers of the right- and left-moving Fourier modes that make up the arbitrary perturbations and $\epsilon_{n}$ and $\epsilon_{m}$ their (small) amplitude to wavelength ratios.

We can use this expression to construct a simple backreaction model. If we split the sum of the wavenumbers in Eq. (1)

$$
\frac{d P}{d l}=\sum_{n} \frac{d P_{n}}{d l}+\sum_{m} \frac{d P_{m}}{d l},
$$

we can identify individual modes with the power they radiate

$$
\frac{d P_{n}}{d l} \sim \pi G \mu^{2} \epsilon_{n}^{2} \kappa_{n} \sum_{m} \epsilon_{m}^{2},
$$

with a similar expression for the $m$ modes travelling in the opposite direction. Each of the modes contributes to the effective mass per unit length of the string by an amount

$$
\delta \mu_{n} \approx \mu \epsilon_{n}^{2}
$$

and the power radiated into gravitational waves by each of the modes decreases this contribution [9]

$$
\frac{d P_{n}}{d l}=-\frac{d}{d t}\left(\delta \mu_{n}\right) .
$$


By putting Eq. (5) together with Eqs. (画) and (34) we arrive at an expression for the time evolution of the amplitude to wavelength ratio of each of the modes

$$
\dot{\epsilon}_{n} \sim-\pi G \mu \kappa_{n} \epsilon_{n} \sum_{m} \epsilon_{m}^{2}
$$

with an analogous expression for the left-moving $m$ modes. Each mode therefore loses amplitude at a rate proportional to its own frequency, to its amplitude to wavelength ratio and to a sum which represents the interaction of the mode with every other mode moving in the opposite direction.

There remains, however, some puzzling behavior. In the case of just two colliding modes we can write Eq. (11) as

$$
\frac{d P}{d l} \sim \pi G \mu^{2}\left(\kappa_{a}+\kappa_{b}\right) \epsilon_{a}^{2} \epsilon_{b}^{2}
$$

where the $a$ and $b$ subscripts differentiate between the right- and left-moving modes. If we conformally stretch (say) the $a$ mode such that $\epsilon_{a}$ remains constant while $\kappa_{a} \rightarrow 0$, Eq. (7) approaches the constant

$$
\frac{d P}{d l} \sim \pi G \mu^{2} \kappa_{b} \epsilon_{a}^{2} \epsilon_{b}^{2} .
$$

This result is in contradiction to the fact that the power vanishes in the case of perturbations travelling in only one direction on a string [11]: By conformally stretching one of the modes we are making the string on which the other mode is travelling straighter and we expect the power radiated to approach zero in that limit.

This problem was investigated in [12]. Here we will summarise the results. It turns out that in order to be in the Hindmarsh regime (the regime where Eq. (7) is valid) it is not merely sufficient for the amplitudes of each of the colliding modes to be smaller than their corresponding wavelengths: It is also necessary for both amplitudes to be small compared to the geometric mean of the wavelengths, namely it is necessary to have

$$
\epsilon_{a}^{2} \frac{\kappa_{b}}{\kappa_{a}}=\frac{A_{a}^{2}}{\lambda_{a} \lambda_{b}} \ll 1
$$

and

$$
\epsilon_{b}^{2} \frac{\kappa_{a}}{\kappa_{b}}=\frac{A_{b}^{2}}{\lambda_{a} \lambda_{b}} \ll 1,
$$

where $A_{a}$ and $A_{b}$ are the amplitudes and $\lambda_{a}$ and $\lambda_{b}$ are the wavelengths of the two colliding modes. If instead we are in the regime where one of these two Lorentz invariant quantities, say,

$$
\epsilon_{a}^{2} \frac{\kappa_{b}}{\kappa_{a}}=\frac{A_{a}^{2}}{\lambda_{a} \lambda_{b}} \gg 1
$$

the power radiated is exponentially suppressed. This is precisely the regime where the power given by Eq. (7) tends to the constant Eq. (8). It was also found that when the quantity $\epsilon_{a}^{2} \kappa_{b} / 8 \kappa_{a}$ increases past 1 the power drops discontinuously.

We can approximate this behaviour by introducing a cutoff in Eq. (6) such that the sum excludes interactions between modes that satisfy

$$
\epsilon_{a}^{2} \frac{\kappa_{b}}{\kappa_{a}}>8
$$

as follows

$$
\dot{\epsilon}_{n} \sim-\pi G \mu \kappa_{n} \epsilon_{n} \sum_{m} \epsilon_{m}^{2} \theta\left(8-\epsilon_{n}^{2} \frac{\kappa_{m}}{\kappa_{n}}\right) \theta\left(8-\epsilon_{m}^{2} \frac{\kappa_{n}}{\kappa_{m}}\right)
$$

with a similar equation for the $m$-modes traveling in the opposite direction. This approximation is justified because of the discontinuous drop in the power that takes place when $\epsilon_{a}^{2} \kappa_{b} / \kappa_{a}=8$ and the exponential suppression that sets in when Eq. (11) is satisfied. In fact we cannot easily associate the power radiated with a given mode outside of the Hindmarsh regime and it is fortunate that in this case the power radiated is exponentially supressed and therefore negligible. It should be noted that the calculation in 12 was performed for just two modes travelling in opposite directions. Here we have assumed (reasonably, we believe) that when a mode interacts with a collection of modes all of which satisfy Eq. (11) the power is also negligible.

Eq. (13) can be used to find the evolution of a set of amplitude to wavelength ratios from some initial conditions. There are two distinct cases of interest. On a long string, one can consider Eq. (13) to give the damping of the average perturbation with a certain wavelength. By symmetry the left-moving and right-moving perturbations will have the same average amplitudes. However, in the case of a loop, a statistical fluctuation may produce an excess of power in a particular direction over a broad range of wavelengths. In that case, since a perturbation passes the same oppositely-directed perturbations over and over again, it is essential to treat the right- and left-movers separately. Here we will consider the case of long strings only.

\section{THE SIZE OF THE SMALLEST SCALES}

When the time-scale over which modes on a long string are significantly stretched by the expansion of the universe, which is about $t$, the cosmic time, is large compared to the gravitational damping time, Eq. (13) can be used to estimate the size of the smallest scales on long strings: The $n$th mode will be significantly damped in amplitude provided $\tau_{g}<t$ with $\tau_{g}$ given by

$$
\frac{\dot{\epsilon}_{n}}{\epsilon_{n}} \sim-\frac{1}{\tau_{g}} .
$$

We expect that a combination of stretching by the expansion of the universe and self-intersections on strings 
will result in an average spectrum of pertubations where the small scales are suppressed relative to the large scales. This seems reasonable even when kinks (produced at intercommutation events) are present since the amplitude to wavelength ratio of Fourier components of kinks as a function of mode number decays like $1 / n$. The presence of a flat or decreasing spectrum means the sum in Eq. (13) is going to be bounded from below and above: A given mode does not see every other mode on the string but rather a range of modes that lie around it. If we assume a power law spectrum

$$
\epsilon_{n} \sim n^{-\beta}
$$

one can use Eq. (12) to show that the lower bound on $m$ for a given mode $n$ is

$$
m_{\mathrm{Min}} \sim\left(\frac{n}{8}\right)^{1 /(1+2 \beta)}
$$

which corresponds to the largest wavelength mode that the $n$th mode interacts with while still in the Hindmarsh regime. The smallest wavelength mode that it interacts with can also be found from Eq. (12) and is given by

$$
m_{\mathrm{Max}} \sim 8 n^{1+2 \beta}
$$

We can therefore write Eq. (13) as

$$
\frac{\dot{\epsilon}_{n}}{\epsilon_{n}} \sim-\pi G \mu \kappa_{n} \sum_{m=m_{\mathrm{Min}}}^{m_{\mathrm{Max}}} \epsilon_{m}^{2}
$$

with $m_{\mathrm{Min}}$ and $m_{\mathrm{Max}}$ given by Eqs. (16) and (17) above.

Previously, it was assumed that all modes interact with all other modes and therefore for spectra where the small scales are sufficiently suppressed, namely those with $\beta>$ $1 / 2$

$$
\sum_{m} \epsilon_{m}^{2} \sim 1
$$

This means that we would expect the survival of modes whose wavelengths

$$
\lambda>2 \pi^{2} G \mu t
$$

independently of the specific spectrum of perturbations present on the string.

In fact we need to evaluate the sum between the two limits. We can approximate the sum in Eq. (18) by an integral

$$
\sum_{m=m_{\mathrm{Min}}}^{m_{\mathrm{Max}}} \epsilon_{m}^{2} \sim \int_{m_{\mathrm{Min}}}^{m_{\mathrm{Max}}} d m m^{-2 \beta}
$$

and take the dominant contribution which when $\beta>1 / 2$ is given by

$$
\int_{m_{\mathrm{Min}}}^{m_{\mathrm{Max}}} d m m^{-2 \beta} \sim-\frac{1}{1-2 \beta} m_{\mathrm{Min}}^{1-2 \beta} .
$$

The dominant contribution therefore comes from the largest wavelength mode that the $n$th mode can interact with.

Using Eq. (22) we can write Eq. (18) as

$$
\frac{\dot{\epsilon}_{n}}{\epsilon_{n}} \sim \frac{1}{1-2 \beta} \pi G \mu \kappa_{n} m_{\mathrm{Min}}{ }^{1-2 \beta} .
$$

Using Eqs. (14) and (16) we can see that

$$
\frac{1}{\tau_{g}} \sim \frac{\Gamma G \mu}{\lambda} n^{(1-2 \beta) /(1+2 \beta)},
$$

where

$$
\Gamma=\frac{8^{(2 \beta-1) /(2 \beta+1)}}{2 \beta-1} 2 \pi^{2} .
$$

Since we want the gravitational lifetime of the mode to be larger than the cosmic time and using $n \sim t / \lambda$,

$$
\frac{\Gamma G \mu}{\lambda}\left(\frac{t}{\lambda}\right)^{(1-2 \beta) /(1+2 \beta)}<\frac{1}{t},
$$

and therefore only modes with wavelengths

$$
\lambda>(\Gamma G \mu)^{(1+2 \beta) / 2} t,
$$

have a significant amplitude at time $t$.

\section{SIMPLE EXAMPLES}

When the amplitude is small compared to the wavelength, one can find the effect of cosmological expansion on the spectrum of perturbations on a string [13]. If the wavelength of a mode on a long string is larger than the horizon, then it is stretched conformally; its amplitude and wavelength grow with the scale factor. If, on the other hand, the wavelength of the mode is smaller then the horizon, only its wavelength is stretched. The net result of this cosmological processing is that the amplitude to wavelength ratio of modes larger than the horizon as a function of the wavenumber remains constant and for modes inside the horizon it becomes a power law.

It is not hard to see why this is so. If a mode enters the horizon at a time $t_{0}$, then its wavelength and amplitude are both $\sim t_{0}$. While inside the horizon the amplitude remains fixed and the wavelength is stretched so that at sometime $t$ later

$$
\lambda_{0}(t) \sim t_{0}\left(\frac{t}{t_{0}}\right)^{\alpha}=t_{0}^{1-\alpha} t^{\alpha}
$$

where $\alpha=1 / 2$ or $2 / 3$ depending on whether we are in the radiation or matter era. We can therefore write the amplitude to wavelength ratio of that mode as

$$
\epsilon_{0}(t) \sim \frac{t_{0}}{t_{0}^{1-\alpha} t^{\alpha}}=\frac{t_{0}^{\alpha}}{t^{\alpha}} .
$$


If we imagine that our mode of wavelength $\lambda_{0}(t)$ is some fraction of the largest mode, $\lambda_{0}(t)=\lambda / n$ with $\lambda \sim t$, it is easy to see that

$$
t_{0}=t n^{-1 /(1-\alpha)}
$$

which when substituted into Eq. (29) yields the spectrum

$$
\epsilon_{n} \sim n^{-\alpha /(1-\alpha)}=\left\{\begin{array}{lr}
n^{-1} & \text { Radiation Era } \\
n^{-2} & \text { Matter Era }
\end{array}\right.
$$

Feeding the spectrum Eq. (31) into Eq. (27) yields

$\lambda>(\Gamma G \mu)^{(1+\alpha) / 2(1-\alpha)} t=\left\{\begin{array}{lc}(\Gamma G \mu)^{3 / 2} t & \text { Radiation Era } \\ (\Gamma G \mu)^{5 / 2} t & \text { Matter Era }\end{array}\right.$

as the small scale structure cutoffs in the radiation and matter eras. However, this analysis neglects intercommutations, which produce kinks, and the possibility that small perturbations propagating on curved horizon-sized strings may not be efficiently stretched.

\section{CONCLUSIONS}

We have presented a method for the calculation of gravitational back-reaction on cosmic strings taking into account that not all Fourier modes that make up the perturbations on strings interact with all other modes with the same efficiency $[12$.

In particular, modes of a given wavelength only interact significantly with a narrow range of other modes whose wavelengths are comparable to it. This range depends on the spectrum of perturbations on the string at late times (when gravitational effects become important) which in turn has the effect of making the small scale structure cutoff sensitive to this spectrum.

Using this method and assuming a power law spectrum we have arrived at an expression for the small scale structure cutoff. We have further applied it to the spectrum resulting from the stretching of small amplitude waves in a radiation- or matter-dominated FRW universe.

It is unclear to what extent the results obtained for these two simple cases apply to a realistic network of cosmic strings because we have ignored the effect of intercommutations, which leads to kinks, as well as the possibility that small perturbations propagating on curved horizon-sized strings may not be significantly stretched by the expansion of the universe. Both of these effects have the potential to change the spectrum of perturbations and therefore the value of the small scale structure cutoff.

However, it is clear that because of the restricted range of interaction affecting every mode, the small scale structure cutoff will be smaller than the one given by the usual calculation where an unrestricted range of interaction is assumed.

\section{ACKNOWLEDGMENTS}

We would like to thank Jose Juan Blanco-Pillado for useful discussions. The work of KDO and AV was partially funded by the NSF.

[1] T.W.B. Kibble, J. Phys. A9 (1976) 1387.

[2] A. Vilenkin and E.P.S. Shellard, Cosmic strings and other Topological Defects. Cambridge University Press, 2000.

[3] D.P. Bennet and F.R. Bouchet, Phys. Rev. Lett. 60 (1988) 257.

[4] V. Berezinski, B. Hnatyk and A. Vilenkin, Phys.Rev. D64 (2001) 043004.

[5] T. Damour and A. Vilenkin, Phys. Rev. Lett. 85(2000) 3761.

[6] B. Allen and A.C. Ottewill, Phys. Rev D63 (2001) 063507.

[7] P. Bhattacharjee and G. Sigl, Phys. Rep. 327 (2000) 109.

[8] V. Berezinsky, P. Blasi and A. Vilenkin, Phys. Rev. D58 (1998) 103515.

[9] M. Hindmarsh, Phys. Lett. B251 (1990) 28.

[10] R.A. Battye and E.P.S.Shellard, Nucl. Phys. B423 (1994) 260.

[11] D. Garfinkle and T. Vachaspati, Phys. Rev. D42 (1990) 1960.

[12] X. Siemens and K.D. Olum, Nucl.Phys. B611 (2001) 125-145.

[13] A. Vilenkin, Phys. Rev D24 (1981) 2082. 